\title{
DISCRETE AHLFORS-BEURLING TRANSFORM AND ITS PROPERTIES
}

\begin{abstract}
The Ahlfors-Beurling transform has been well studied on classical Lebesgue, Morrey, Sobolev, Besov, Campanato, etc. spaces. However, its discrete version is still not studied well. In this paper, we study the properties of the discrete Ahlfors-Beurling transform on discrete Lebesgue spaces.
\end{abstract}

Key words: discrete Ahlfors-Beurling transform, Lebesgue spaces, boundedness

2010 Mathematical Subject Classification: 44A15, 40A05, 46B45, 42B35

1. Introduction. The Ahlfors-Beurling transform of a function $f \in L_{p}(C), 1 \leq p<\infty$, is defined as the following singular integral:

$$
(B f)(z)=-\frac{1}{\pi} \lim _{\varepsilon \rightarrow 0} \int_{\{w \in C:|z-w|>\varepsilon\}} \frac{f(w)}{(z-w)^{2}} d m(w) .
$$

The Ahlfors-Beurling transform is one of the important operators in complex analysis. It has been shown in [1], [5], [8], [13], [20] that this transform plays an essential role in applications to the theory of quasiconformal mappings and to the Beltrami equation with discontinuous coefficients.

From the theory of singular integrals (see [7], [16]), it is known that the Ahlfors-Beurling transform is a bounded operator in the space $L_{p}$, $1<p<\infty$; that is, if $f \in L_{p}$, then $B f \in L_{p}$ and the inequality

$$
\|B f\|_{L_{p}} \leq C_{p}\|f\|_{L_{p}}
$$

holds. In the case $f \in L_{1}$, only the weak inequality holds:

$$
m\{z \in C:|(B f)(z)|>\lambda\} \leq \frac{C_{1}}{\lambda}\|f\|_{L_{1}}, \quad \lambda>0,
$$

(C) Petrozavodsk State University, 2020 
where $m$ stands for the Lebesgue measure, $C_{p}, C_{1}$ are constants independent of $f$.

Note that the Ahlfors-Beurling transform of a function $f \in L_{1}$ is not Lebesgue integrable. In [4], the authors considered a modified version of the Ahlfors-Beurling transform and, using the notion of $A$-integrability, proved an analogue of the Riesz equality.

In [6], [9], [10], [11], [12], [14], [18], [19], the boundedness of the operator $B$ in other function spaces (in the spaces of Sobolev, Besov, Campanato, Morrey, etc.) was studied. But the discrete version of the AhlforsBeurling transform has not been studied. In this paper, we study the properties of the discrete Ahlfors-Beurling transform on discrete Lebesgue spaces.

2. Discrete Ahlfors-Beurling transform and its boundedness on discrete Lebesgue spaces.

Denote by $l_{p}:=l_{p}\left(Z_{C}\right), p \geq 1$, the class of sequences $h=\left\{h_{n}\right\}_{n \in Z_{C}}$ satisfying the condition

$$
\|h\|_{l_{p}}:=\left(\sum_{n \in Z_{C}}\left|h_{n}\right|^{p}\right)^{1 / p}<\infty
$$

where $Z_{C}:=\{m+i n \in C: m, n \in Z\}$ and $Z$ is the set of integers.

Let $h=\left\{h_{n}\right\}_{n \in Z_{C}} \in l_{p}, p \geq 1$. Namely, the sequence $\tilde{B}(h)=\left\{(\tilde{B} h)_{n}\right\}_{n \in Z_{C}}$ is called the Ahlfors-Beurling transform of the sequence $h$, where

$$
(\tilde{B} h)_{n}=\sum_{m \in Z_{C}, m \neq n} \frac{h_{m}}{(n-m)^{2}}, \quad n \in Z_{C} .
$$

Note that if $h \in l_{p}, 1 \leq p<\infty$, then from the Holder inequality it follows that the series $\sum_{m \in Z_{C}, m \neq n} \frac{h_{m}}{(n-m)^{2}}$ absolutely converges, and, therefore, the Ahlfors-Beurling transform of the sequence $h$ exists. In [7], A.P. Calderon and A. Zygmund noted that the discrete Ahlfors-Beurling transform is of special interest among discrete analogues of singular integrals. In this work, it was also noted (without proof) that the discrete analogues of singular integrals, including the discrete Ahlfors-Beurling transform, is bounded in $L_{p}$. For $n=1$, this remark is due to M. Riesz [15] (see also [17]), and the proof in the case of general $n$ follows a similar pattern. For the sake of readability of the article, we first give a proof of this fact. 
Theorem 1. Let $1<p<\infty$. For any $h \in l_{p}$, we have $\tilde{B} h \in l_{p}$, and there exists $c_{p}>0$ such that

$$
\|\tilde{B} h\|_{l_{p}} \leq c_{p} \cdot\|h\|_{l_{p}}
$$

holds for all $h \in l_{p}$.

Proof. Define the function $f(z)$ to be $\left(-4 \pi h_{n}\right)$ for $z \in P(n, 1 / 4), n \in Z_{C}$, and 0 elsewhere, where

$$
P(n, \delta):=\{w \in C:-\delta \leq \Re(w-n)<\delta,-\delta \leq \Im(w-n)<\delta\} .
$$

It follows from $h \in l_{p}$, that $f \in L_{p}$ and

$$
\|f\|_{L_{p}}=\left(\sum_{n \in Z_{C}} \int_{P(n, 1 / 4)}\left|4 \pi h_{n}\right|^{p} d m(z)\right)^{1 / p}=4^{1-1 / p} \pi\|h\|_{l_{p}} .
$$

Then, from inequality (1) it follows that $B f \in L_{p}$, and

$$
\|B f\|_{L_{p}} \leq C_{p} 4^{1-1 / p} \pi\|h\|_{l_{p}} .
$$

Define the function $F(z)$ to be $(\tilde{B} h)_{n}$ for $z \in P(n, 1 / 2), n \in Z_{C}$ and

$$
G(z)=(B f)(z)-F(z) .
$$

We first prove that $G(z) \in L_{p}$.

For every $z \in P(n, 1 / 2),|\Re(z-n)| \neq 1 / 4,|\Im(z-n)| \neq 1 / 4, n \in Z_{C}$, we have

$$
\begin{aligned}
G(z)= & \sum_{m \in Z_{C}} 4 h_{m} \int_{P(m, 1 / 4)} \frac{d m(w)}{(z-w)^{2}}-\sum_{m \in Z_{C}, m \neq n} \frac{h_{m}}{(n-m)^{2}}= \\
= & \sum_{m \in Z_{C}, m \neq n} 4 h_{m} \int_{P(m, 1 / 4)}\left(\frac{1}{(z-w)^{2}}-\frac{1}{(n-m)^{2}}\right) d m(w)+ \\
& +4 h_{n} \int_{P(n, 1 / 4)} \frac{d m(w)}{(z-w)^{2}}=G_{1}(z)+G_{2}(z),
\end{aligned}
$$

where

$$
\int_{P(n, 1 / 4)} \frac{d m(w)}{(z-w)^{2}}:=\lim _{\varepsilon \rightarrow 0+} \int_{\{w \in P(n, 1 / 4):|w-z| \geq \varepsilon\}} \frac{d m(w)}{(z-w)^{2}} .
$$


Let $m \neq n$. Since for every $z \in P(n, 1 / 2)$ and $w \in P(m, 1 / 4)$

$$
|n-m|-3 / 4 \leq|z-w| \leq|n-m|+3 / 4
$$

then we get

$$
\begin{gathered}
\left|\frac{1}{(z-w)^{2}}-\frac{1}{(n-m)^{2}}\right|=\frac{|n-m-z+w| \cdot|n-m+z-w|}{|z-w|^{2} \cdot|n-m|^{2}} \leq \\
\quad \leq \frac{3 / 4(2|n-m|+3 / 4)}{|n-m|^{2} \cdot(|n-m|-3 / 4)^{2}} \leq \frac{33}{|n-m|^{3}}
\end{gathered}
$$

Therefore, for every $z \in P(n, 1 / 2)$

$$
\begin{aligned}
\left|G_{1}(z)\right| \leq \sum_{m \in Z_{C}, m \neq n} 4\left|h_{m}\right| \int_{P(m, 1 / 4)} \mid \frac{1}{(z-w)^{2}} & -\frac{1}{(n-m)^{2}} \mid d m(w) \leq \\
& \leq \sum_{m \in Z_{C}, m \neq n} \frac{33\left|h_{m}\right|}{|n-m|^{3}}
\end{aligned}
$$

From this and from the Holder inequality, it follows that

$$
\begin{gathered}
\left\|G_{1}\right\|_{L_{p}} \leq 33\left(\sum_{n \in Z_{C}}\left[\sum_{m \in Z_{C}, m \neq n} \frac{\left|h_{m}\right|}{|n-m|^{3}}\right]^{p}\right)^{1 / p} \leq \\
\leq 33\left(\sum_{n \in Z_{C}}\left[\sum_{m \in Z_{C}, m \neq n} \frac{\left|h_{m}\right|^{p}}{|n-m|^{3}}\right] \cdot\left[\sum_{m \in Z_{C}, m \neq n} \frac{1}{|n-m|^{3}}\right]^{p-1}\right)^{1 / p}= \\
=33 d_{0}^{1-1 / p}\left(\sum_{n \in Z_{C}} \sum_{m \in Z_{C}, m \neq n} \frac{\left|h_{m}\right|^{p}}{|n-m|^{3}}\right)^{1 / p}= \\
=33 d_{0}^{1-1 / p}\left(\sum_{m \in Z_{C}}\left|h_{m}\right|^{p} \sum_{n \in Z_{C}, n \neq m} \frac{1}{|n-m|^{3}}\right)^{1 / p}=33 d_{0}\|h\|_{l_{p}},
\end{gathered}
$$

where $d_{0}=\sum_{n \in Z_{C}, n \neq 0} \frac{1}{|n|^{3}}$.

Let us show that $G_{2} \in L_{p}$. If $z \in P(n, 1 / 2),|\Re(z-n)| \neq 1 / 4,|\Im(z-n)| \neq$ $1 / 4, n \in Z_{C}$, then

$$
\left|G_{2}(z)\right| \leq 4\left|h_{n}\right| \int_{U(n, 2) \backslash U(n, \rho(z))} \frac{d m(w)}{|z-w|^{2}}=8 \pi\left|h_{n}\right| \cdot \ln \frac{2}{\rho(z)},
$$


where $\rho(z):=\rho(z ; \overline{P(n, 1 / 4)})=\min \{|z-w|: w \in \overline{P(n, 1 / 4)}\}$ is the distance

from the point $z$ to the set $\overline{P(n, 1 / 4)}$ and $U(z, \varepsilon)=\{w \in C:|w-z|<\varepsilon\}$. Therefore, for every $n \in Z_{C}$,

$$
\int_{P(n, 1 / 2)}\left|G_{2}(z)\right|^{p} d m(z) \leq(8 \pi)^{p}\left|h_{n}\right|^{p} \cdot \int_{P(n, 1 / 2)}\left(\ln \frac{2}{\rho(z)}\right)^{p} d m(z)=
$$

$$
=(8 \pi)^{p}\left|h_{n}\right|^{p} \cdot 8 \int_{0}^{1 / 2} d x \int_{x}^{1 / 2}\left(\ln \frac{2}{1 / 2-y}\right)^{p} d y \leq(8 \pi)^{p}\left|h_{n}\right|^{p} \cdot 4 d_{1},
$$

where $d_{1}:=\int_{0}^{1 / 2}\left(\ln \frac{2}{1 / 2-y}\right)^{p} d y$.

From this, we have

$$
\left\|G_{2}\right\|_{L_{p}}=\left(\sum_{n \in Z_{C}} \int_{P(n, 1 / 2)}\left|G_{1}(z)\right|^{p} d m(z)\right)^{1 / p} \leq 8 \pi \cdot\left(4 d_{1}\right)^{1 / p}\|h\|_{l_{p}}
$$

It follows from (4), (6), (8), that $G \in L_{p}$.

Since $F(z)=(B f)(z)-G(z)$, then we have from $B f \in L_{p}$ and $G \in L_{p}$ that $F \in L_{p}$,

$$
\|F\|_{L_{p}} \leq\left(C_{p} 4^{1-1 / p} \pi+33 d_{0}+8 \pi \cdot\left(4 d_{1}\right)^{1 / p}\right)\|b\|_{l_{p}} .
$$

Therefore,

$$
\begin{aligned}
\|\tilde{B} h\|_{l_{p}}=\left(\sum_{n \in Z_{C}}\left|(\tilde{B} h)_{n}\right|^{p}\right)^{1 / p} & =\left(\sum_{n \in Z_{C}} \int_{P(n, 1 / 2)}|F(z)|^{p} d m(z)\right)^{1 / p}= \\
=\|F\|_{L_{p}} & \leq\left(C_{p} 4^{1-1 / p} \pi+33 d_{0}+8 \pi \cdot\left(4 d_{1}\right)^{1 / p}\right)\|b\|_{l_{p}} .
\end{aligned}
$$

This completes the proof of Theorem.

Theorem 2. There exists $c_{1}>0$, such that for any $h \in l_{1}$ and for any $\lambda>0$ the distribution function $(\tilde{B} h)(\lambda)=\left|\left\{n \in Z_{C}:\left|(\tilde{B} h)_{n}\right|>\lambda\right\}\right|:=$ $\sum_{\left\{n \in Z_{C}:\left|(\tilde{B} h)_{n}\right|>\lambda\right\}} 1$ of the Ahlfors-Beurling transform of the sequence $h$ satisfies the inequality

$$
|(\tilde{B} h)(\lambda)| \leq \frac{c_{1}}{\lambda}\|h\|_{l_{1}}
$$


Proof. We define the functions $f(z), F(z), G(z), G_{1}(z), G_{2}(z)$ as in the proof of Theorem 1. It follows from $h \in l_{1}$ that $f \in L_{1}$,

$$
\|f\|_{L_{1}}=\sum_{n \in Z_{C}} \int_{P(n, 1 / 4)}\left|4 \pi h_{n}\right| d m(z)=\pi\|h\|_{l_{1}} .
$$

Then, from (2) we have

$$
m\{z \in C:|(B f)(z)|>\lambda\} \leq \frac{C_{1}}{\lambda} \pi\|h\|_{l_{1}}, \lambda>0 .
$$

It follows from (5) and (7) that $G_{1} \in L_{1}$ and $G_{2} \in L_{1}$,

$$
\begin{gathered}
\left\|G_{1}\right\|_{L_{1}}=\sum_{n \in Z_{C}} \int_{P(n, 1 / 2)}\left|G_{1}(z)\right| d m(z) \leq 33 \sum_{n \in Z_{C}} \sum_{m \in Z_{C}, m \neq n} \frac{\left|h_{m}\right|}{|n-m|^{3}}= \\
=33 \sum_{m \in Z_{C}}\left|h_{m}\right| \sum_{n \in Z_{C}, n \neq m} \frac{1}{|n-m|^{3}}=33 d_{0}\|h\|_{l_{1}}, \\
\left\|G_{2}\right\|_{L_{1}}=\int_{C}\left|G_{2}(z)\right| d m(z) \leq 8 \pi \cdot \sum_{n \in Z_{C}}\left|h_{n}\right| \int_{P(n, 1 / 2)} \ln \frac{2}{\rho(z)} d m(z) \leq \\
\leq 32 \pi \int_{0}^{1 / 2} \ln \frac{2}{1 / 2-y} d y \cdot\|h\|_{l_{1}}=16 \pi[\ln 4+1] \cdot\|h\|_{l_{1}} .
\end{gathered}
$$

It follows from (4), (11), (12) that $G \in L_{1}$,

$$
\|G\|_{L_{1}} \leq\left[16 \pi(\ln 4+1)+33 d_{0}\right] \cdot\|h\|_{l_{1}} .
$$

Therefore, by Chebyshev's inequality, we have

$$
m\{z \in C:|G(z)|>\lambda\} \leq \frac{16 \pi(\ln 4+1)+33 d_{0}}{\lambda}\|h\|_{l_{1}} .
$$

Since $F(z)=(B f)(z)-G(z)$, then it follows from (10) and (13) that

$$
\begin{aligned}
\{z \in C & :|F(z)|>\lambda\} \leq m\left\{z \in C:|(B f)(z)|>\frac{\lambda}{2}\right\}+ \\
& +m\left\{z \in C:|G(z)|>\frac{\lambda}{2}\right\} \leq \frac{2 C_{1} \pi+32 \pi(\ln 4+1)+66 d_{0}}{\lambda}\|h\|_{l_{1}} .
\end{aligned}
$$


Therefore,

$$
\begin{aligned}
(\tilde{B} h)(\lambda)=\mid\left\{n \in Z_{C}:\left|(\tilde{B} h)_{n}\right|>\right. & \lambda\} \mid=m\{z \in C:|F(z)|>\lambda\} \leq \\
& \leq \frac{2 C_{1} \pi+32 \pi(\ln 4+1)+66 d_{0}}{\lambda}\|h\|_{l_{1}} .
\end{aligned}
$$

This completes the proof.

3. Asymptotic behavior of the distribution function of the discrete Ahlfors-Beurling transform.

The following theorem shows the main reason why the discrete AhlforsBeurling transform does not belong to the class $l_{1}$.

Theorem 3. Let $h \in l_{1}$. Then the equation

$$
\lim _{\lambda \rightarrow 0+} \lambda \cdot(\tilde{B} h)(\lambda)=\pi\left|\sum_{n \in Z_{C}} h_{n}\right|
$$

holds.

At first, we prove the auxiliary lemma.

Lemma 1. Let $h \in l_{1}$ and $\sum_{n \in Z_{C}} h_{n}=0$. Then the equation

$$
(\tilde{B} h)(\lambda)=o(1 / \lambda), \lambda \rightarrow 0+
$$

holds.

Proof. At first, assume that the sequence $h \in l_{1}$ is concentrated on some finite ball $\{w \in C:|w| \leq m\}$, that is, $h_{n}=0$ for $|n|>m$. In this case, from the equality

$$
(\tilde{B} h)_{n}=\sum_{|k| \leq m} \frac{h_{k}}{(n-k)^{2}}-\frac{1}{n^{2}} \sum_{|k| \leq m} h_{k}=\sum_{|k| \leq m} \frac{2 n-k}{(n-k)^{2} n^{2}} k h_{k},|n|>m
$$

we get that

$$
\left|(\tilde{B} h)_{n}\right| \leq \frac{8}{|n|^{3}} \sum_{|k| \leq m}\left|k h_{k}\right|
$$

for large values of $n$, whence it follows asymptotic equation (15).

Now let us consider the general case. From the condition $\sum_{n \in Z_{C}} h_{n}=0$ it follows that for any $\varepsilon>0$ there exist the sequences $h^{\prime}=\left\{h_{n}^{\prime}\right\}_{n \in Z} \in$ $l_{1}$ and $h^{\prime \prime}=\left\{h_{n}^{\prime \prime}\right\}_{n \in Z} \in l_{1}$ satisfying the condition: $h=h^{\prime}+h^{\prime \prime}$; the 
sequence $h^{\prime} \in l_{1}$ is concentrated on some finite ball $\{w \in C:|w| \leq m\}$ and $\sum_{n \in Z_{C}} h_{n}^{\prime}=0 ; h^{\prime \prime} \in l_{1}$ satisfies the inequality $\sum_{n \in Z_{C}}\left|h_{n}^{\prime \prime}\right|<\frac{\varepsilon}{4 c_{1}}$, where $c_{1}$ is the constant in estimation (9). Since the sequence $h^{\prime} \in l_{1}$ is concentrated on $\{w \in C:|w| \leq m\}$ and $\sum_{n \in Z_{C}} h_{n}^{\prime}=0$, then for the sequence $h^{\prime} \in l_{1}$ equation (15) is satisfied, and, therefore, there exists $\lambda(\varepsilon)>0$ such that for $0<\lambda<\lambda(\varepsilon)$ the inequality

$$
\lambda\left(\tilde{B} h^{\prime}\right)(\lambda / 2)<\varepsilon / 2
$$

holds, where $\left(\tilde{B} h^{\prime}\right)(\lambda)=\sum_{\left\{n \in Z_{C}:\left|\left(\tilde{B} h^{\prime}\right)_{n}\right|>\lambda\right\}} 1$. On the other hand, from inequality (9) it follows that for any $\lambda>0$

$$
\lambda\left(\tilde{B} h^{\prime \prime}\right)(\lambda / 2)<2 c_{1}\left\|h^{\prime \prime}\right\|_{l_{1}}<\varepsilon / 2
$$

where $\left(\tilde{B} h^{\prime \prime}\right)(\lambda)=\sum_{\left\{n \in Z_{C}:\left|\left(\tilde{B} h^{\prime \prime}\right)_{n}\right|>\lambda\right\}} 1$. From inequalities (16), (17) and from the inclusion

$$
\begin{aligned}
& \left\{n \in Z_{C}:\left|(\tilde{B} h)_{n}\right|>\lambda\right\} \subset \\
& \quad \subset\left\{n \in Z_{C}:\left|\left(\tilde{B} h^{\prime}\right)_{n}\right|>\lambda / 2\right\} \cup\left\{n \in Z_{C}:\left|\left(\tilde{B} h^{\prime \prime}\right)_{n}\right|>\lambda / 2\right\}
\end{aligned}
$$

we get

$$
\lambda \cdot(\tilde{B} h)(\lambda) \leq \lambda\left(\tilde{B} h^{\prime}\right)(\lambda / 2)+\lambda\left(\tilde{B} h^{\prime \prime}\right)(\lambda / 2)<\varepsilon
$$

for $0<\lambda<\lambda(\varepsilon)$. This shows that equality (15) was satisfied for all $h \in l_{1}$ satisfying a condition $\sum_{n \in Z_{C}} h_{n}=0$. This completes the proof of the lemma.

Proof of theorem 3. In the case $\sum_{n \in Z_{C}} h_{n}=0$ the assertion of the theorem follows from Lemma 1. Let us consider the case $\sum_{n \in Z_{C}} h_{n}=\alpha \neq 0$. Denote by $h_{n}^{\prime}=h_{n}$ for $n \neq 0, h_{0}^{\prime}=h_{0}-\alpha$ and $h_{n}^{\prime \prime}=0$ for $n \neq 0, h_{0}^{\prime \prime}=\alpha$. Then $h=h^{\prime}+h^{\prime \prime}$, where $h^{\prime}=\left\{h_{n}^{\prime}\right\}_{n \in Z} \in l_{1}$ and $h^{\prime \prime}=\left\{h_{n}^{\prime \prime}\right\}_{n \in Z} \in l_{1}$. Since $\sum_{n \in Z_{C}} h_{n}^{\prime}=0$, then from Lemma 1 we get

$$
\left(\tilde{B} h^{\prime}\right)(\lambda)=o(1 / \lambda), \lambda \rightarrow 0+.
$$

Since $\left(\tilde{B} h^{\prime \prime}\right)_{n}=\frac{\alpha}{n^{2}}$ for $n \neq 0,\left(\tilde{B} h^{\prime \prime}\right)_{0}=0$, then

$$
\left(\tilde{B} h^{\prime \prime}\right)(\lambda) \sim \frac{\pi|\alpha|}{\lambda}, \lambda \rightarrow 0+.
$$


For any $0<\varepsilon<1$, by the inclusions

$$
\begin{aligned}
&\left\{n \in Z_{C}:\left|\left(\tilde{B} h^{\prime \prime}\right)_{n}\right|>\right.(1+\varepsilon) \lambda\} \backslash\left\{n \in Z_{C}:\left|\left(\tilde{B} h^{\prime}\right)_{n}\right|>\varepsilon \lambda\right\} \subset \\
& \subset\left\{n \in Z_{C}:\left|(\tilde{B} h)_{n}\right|>\lambda\right\} \subset \\
& \subset\left\{n \in Z_{C}:\left|\left(\tilde{B} h^{\prime}\right)_{n}\right|>\varepsilon \lambda\right\} \cup\left\{n \in Z_{C}:\left|\left(\tilde{B} h^{\prime \prime}\right)_{n}\right|>(1-\varepsilon) \lambda\right\}
\end{aligned}
$$

and from (18), (19) we have

$$
\frac{\pi|\alpha|}{1+\varepsilon} \leq \liminf _{\lambda \rightarrow 0+} \lambda \cdot(\tilde{B} h)(\lambda) \leq \limsup _{\lambda \rightarrow 0+} \lambda \cdot(\tilde{B} h)(\lambda) \leq \frac{\pi|\alpha|}{1-\varepsilon} .
$$

This implies the equation (14) and completes the proof of Theorem 3.

Note that for the discrete Hilbert transform an analogue of this theorem was proved in [2].

4. A necessary condition and a sufficient condition for the summability of the discrete Ahlfors-Beurling transform.

Theorem 4. Let $h \in l_{1}$. Then, to include $\tilde{B} h \in l_{1}$, it is necessary that the equation

$$
\sum_{n \in Z_{C}} h_{n}=0
$$

holds.

Proof. First, we prove that if the sequence $b=\left\{b_{n}\right\}_{n \in Z} \in l_{1}$, then the distribution function $b(\lambda)=\left|\left\{n \in Z_{C}:\left|b_{n}\right|>\lambda\right\}\right|$ of the sequence $b$ satisfies the condition

$$
b(\lambda)=o(1 / \lambda), \lambda \rightarrow 0+.
$$

It follows, from the inequality

$$
\begin{gathered}
\sum_{n \in Z}\left|b_{n}\right|=\sum_{\left\{n \in Z_{C}:\left|b_{n}\right|>1\right\}}\left|b_{n}\right|+\sum_{k=0}^{\infty}\left[\sum_{\left\{n \in Z_{C}:\left|b_{n}\right| \in\left(2^{-k-1} ; 2^{-k}\right]\right\}}\left|b_{n}\right|\right] \geq \\
\geq\left|\left\{n \in Z_{C}:\left|b_{n}\right|>1\right\}\right|+\sum_{k=0}^{\infty}\left[2^{-k-1} \cdot\left|\left\{n \in Z_{C}:\left|b_{n}\right| \in\left(2^{-k-1} ; 2^{-k}\right]\right\}\right|\right]= \\
=b(1)+\sum_{k=0}^{\infty}\left[2^{-k-1} \cdot\left(b\left(2^{-k-1}\right)-b\left(2^{-k}\right)\right)\right]=\sum_{k=0}^{\infty}\left[2^{-k-1} \cdot b\left(2^{-k}\right)\right]
\end{gathered}
$$


that

$$
\lim _{k \rightarrow \infty} 2^{-k} \cdot b\left(2^{-k}\right)=0 .
$$

Taking into account that the function $b(\lambda)$ is decreasing, we obtain (21).

It follows from $(21)$ that, if $\tilde{B} h \in l_{1}$, then

$$
(\tilde{B} h)(\lambda)=o(1 / \lambda), \lambda \rightarrow 0+,
$$

and, therefore, by Theorem 3, we obtain that equation (20) holds. The proof of the theorem is complete.

Theorem 5. If the sequence $h \in l_{1}$ satisfies the conditions

$$
\begin{aligned}
& \text { i) } \sum_{n \in Z_{C}} h_{n}=0 ; \\
& \text { ii) } \sum_{m \in Z_{C}}\left|h_{m}\right| \ln (e+|m|)<\infty,
\end{aligned}
$$

then $\tilde{B} h \in l_{1}$ and the inequality

$$
\|\tilde{B} h\|_{l_{1}} \leq 150 \sum_{m \in Z_{C}}\left|h_{m}\right| \ln (e+|m|)
$$

holds.

Proof. From the definition of the discrete Ahlfors-Beurling transform, it follows that

$$
\left|(\tilde{B} h)_{0}\right|=\left|\sum_{m \neq 0} \frac{h_{m}}{m^{2}}\right| \leq\|h\|_{l_{1}} .
$$

From condition i) for $n \neq 0$, we have

$$
\begin{aligned}
& \left|(\tilde{B} h)_{n}\right|=\left|\sum_{m \in Z_{C}, m \neq n} \frac{h_{m}}{(n-m)^{2}}-\sum_{m \in Z_{C}} \frac{h_{m}}{n^{2}}\right| \leq \\
& \leq\left|\frac{h_{n}}{n^{2}}\right|+\sum_{m \in Z_{C}, m \neq n}\left|\frac{h_{m}}{(n-m)^{2}}-\frac{h_{m}}{n^{2}}\right| .
\end{aligned}
$$

It follows from inequalities (23) and (24) that

$$
\begin{aligned}
\|\tilde{B} h\|_{l_{1}} & =\sum_{n \in Z_{C}}\left|(\tilde{B} h)_{n}\right| \leq 2\|h\|_{l_{1}}+\sum_{n \in Z_{C}, n \neq 0} \sum_{m \in Z_{C}, m \neq n}\left|\frac{h_{m}}{(n-m)^{2}}-\frac{h_{m}}{n^{2}}\right|= \\
& =2\|h\|_{l_{1}}+\sum_{m \in Z_{C}, m \neq 0}\left|h_{m}\right| \sum_{n \in Z_{C}, n \neq 0, n \neq m}\left|\frac{1}{(n-m)^{2}}-\frac{1}{n^{2}}\right|=
\end{aligned}
$$




$$
=2\|h\|_{l_{1}}+\sum_{m \in Z_{C}, m \neq 0}\left|h_{m}\right| \cdot J_{m},
$$

where

$$
\begin{array}{r}
J_{m}=\sum_{|n| \leq 3|m|, n \neq 0, n \neq m}\left|\frac{1}{(n-m)^{2}}-\frac{1}{n^{2}}\right|+\sum_{|n|>3|m|}\left|\frac{1}{(n-m)^{2}}-\frac{1}{n^{2}}\right|= \\
=J_{m}^{(1)}+J_{m}^{(2)}, \quad m \neq 0 .
\end{array}
$$

Estimate the summands $J_{m}^{(i)}, m \neq 0, i=1,2$. Defining $k=\left[\log _{2}(4|m|)\right]+1$, where $\left[\log _{2}(4|m|)\right]$ is the integer part of the number $\log _{2}(4|m|)$, we have

$$
\begin{gathered}
J_{m}^{(1)} \leq \sum_{|n| \leq 3|m|, n \neq m} \frac{1}{|n-m|^{2}}+\sum_{|n| \leq 3|m|, n \neq 0} \frac{1}{|n|^{2}} \leq \\
\leq 2 \sum_{|n| \leq 4|m|, n \neq 0} \frac{1}{|n|^{2}} \leq 2 \sum_{p=1}^{k} \sum_{2^{p-1} \leq|n|<2^{p}} \frac{1}{|n|^{2}} \leq 2 \sum_{p=1}^{k} 4 \cdot 2^{2 p} \cdot \frac{1}{2^{2 p-2}}=32 k \leq 96 \ln (e+|m|), \\
J_{m}^{(2)}=\sum_{|n|>3|m|} \frac{|m||2 n-m|}{|n-m|^{2}|n|^{2}} \leq 6|m| \sum_{|n|>3|m|} \frac{1}{|n|^{3}}= \\
=6|m| \sum_{p=1}^{\infty} \sum_{3|m| 2^{p-1}<|n| \leq 3|m|^{p}} \frac{1}{|n|^{3}} \leq \\
\leq 6|m| \sum_{p=1}^{\infty} \frac{4 \cdot\left(3|m| \cdot 2^{p}\right)^{2}}{27|m|^{3} \cdot 2^{3 p-3}}=64 \leq 52 \ln (e+|m|) .
\end{gathered}
$$

From this and from (25), (26), we obtain (22). The proof of the theorem is complete.

Note that analogues of these theorems were proved for the discrete Hilbert transform in [3].

Acknowledgments. The authors are very grateful to the referees for many useful comments and suggestions that improved the original manuscript.

\section{References}

[1] Ahlfors, L. V. Lectures on Quasiconformal Mappings. 2nd ed. - University Lecture Series, v. 38. AMS, Providence, RI, 2006. 
[2] Aliev, R. A., Amrahova A. F. Properties of the discrete Hilbert transform. Complex Analysis and Operator Theory. 2019, vol. 13, pp. 3883-3897. DOI: https://doi.org/10.1007/s11785-019-00936-9

[3] Aliev R. A., Amrahova A. F. On the summability of the discrete Hilbert transform. Ural Math. J. 2018, vol. 4, no. 2, pp. 6-12.

DOI: https://doi.org/10.15826/umj .2018.2.002

[4] Aliev R. A., Nabiyeva Kh. I. The A-integral and restricted Ahlfors-Beurling transform. Integral Transforms and Special Functions. 2018, vol. 29, no 10, pp. 820 -830. DOI: https://doi.org/10.1080/10652469.2018.1501046

[5] Astala K., Iwaniec T., Martin G. Elliptic partial differential equations and quasiconformal mappings in the plane. Princeton: University Press, 2009.

[6] Banuelos R., Janakiraman P. L ${ }^{p}$-bounds for the Beurling-Ahlfors transform. Trans. Amer. Math. Sos. 2008, vol. 360, no. 7, pp. 3603-3612.

DOI: https://doi.org/10.1090/S0002-9947-08-04537-6

[7] Calderon, A. P., Zygmund A. On the existence of certain singular integrals. Acta Mathematica, 1952, vol. 88, pp. 85-139.

DOI: https://doi.org/10.1007/BF02392130

[8] Cruz V., Mateu J., Orobitg J. Beltrami equation with coefficient in Sobolev and Besov spaces. Canadian J. Math. 2013, vol. 65, no. 6, pp. 1217-1235. DOI: https://doi.org/10.4153/CJM-2013-001-7

[9] Cruz V., Tolsa X. Smoothness of the Beurling transform in Lipschitz domains. J. Func. Anal. 2012, vol. 262, no. 10, pp. 4423-4457.

DOI: https://doi.org/10.1016/j.jfa.2012.02.023

[10] Doubtsov E., Vasin A. V. Restricted Beurling transforms on Campanato spaces. Complex Variables and Elliptic Eq. 2017, vol. 62, no. 3, pp.333-346. DOI: https://doi.org/10.1080/17476933.2016.1220000

[11] Dragicevic O. Weighted estimates for powers of the Ahlfors-Beurling operators. Proc. Amer. Math. Sos. 2011, vol. 139, no. 6, pp. 2113-2120.

DOI: https://doi.org/10.1090/S0002-9939-2010-10645-7

[12] Kwok-Pun H. The Ahlfors-Beurling transform on Morrey spaces with variable exponents. Integral Transforms and Special Functions. 2018, vol. 29, no. 3 , pp. $207-220$.

DOI: https://doi.org/10.1080/10652469.20137.1422498

[13] Mateu J., Orobitg J., Verdera J. Extra cancellation of even CalderonZygmund operators and quasiconformal mappings. J. Math. Pures et Appl. 2009, vol. 91, no. 4, pp. $402-431$.

DOI: https://doi.org/10.1016/j.matpur.2009.01.010 
[14] Prats M. $L^{p}$-bounds for the Beurling-Ahlfors transform. Publicacions Mat. 2017, vol. 61, no. 2, pp. $291-336$.

DOI: https://doi.org/10.5565/PUBLMAT6121701

[15] Riesz M. Sur les fonctions conjuguees. Mathematische Zeitschrift, 1928, 27, pp. 218-244. DOI: https://doi.org/10.1007/BF01171098

[16] Stein E.M. Singular Integrals and Differentiability Properties of Functions. Princeton: University Press, 1970.

[17] Titchmarsh E. C. Reciprocal formulae involving series and integrals. Math. Z. 1926, vol. 25, pp. $321-347$.

DOI: https://doi .org/10.1007/BF01283842

[18] Tolsa X. Regularity of $C^{1}$ and Lipschitz domains in terms of the Beurling transform. J. Math. Pures et Appl. 2013, vol. 100, pp. 137-165.

DOI: https://doi.org/10.1016/j.matpur.2012.10.014

[19] Vasin A.V. Regularity of the Beurling Transform in Smooth Domains. J. Math. Sciences. 2016, vol. 215, no. 5, pp. 577-584.

[20] Vekua I.N. Generalized analytic functions. - Pergamon Press, 1962.

Received December 06, 2019.

In revised form, April 05, 2020.

Accepted April 06, 2020.

Published online April 13, 2020.

Rashid A. Aliev

Baku State University, Baku, AZ 1148, Azerbaijan

Institute of Mathematics and Mechanics, NAS of Azerbaijan, Baku, AZ 1141, Azerbaijan

E-mail: aliyevrashid@mail.ru

Aynur N. Ahmadova

Sumgait State University, Sumgait, AZ 5008, Azerbaijan

E-mail: dissertant.aynur@gmail.com 